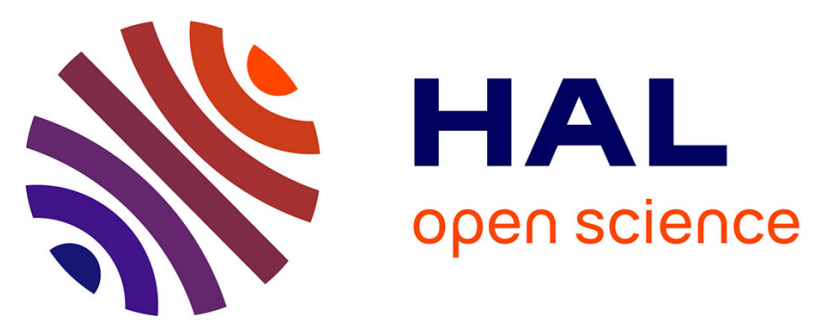

\title{
Modifications superficielles de films de dioxyde d'étain créés sous forte polarisation anodique et au potentiel d'abandon en milieux chlorurés
}

Hubert Cachet, Guy Folcher, Sanae Haskouri, Bernard Tribollet, Dominique Festy

\section{To cite this version:}

Hubert Cachet, Guy Folcher, Sanae Haskouri, Bernard Tribollet, Dominique Festy. Modifications superficielles de films de dioxyde d'étain créés sous forte polarisation anodique et au potentiel d'abandon en milieux chlorurés. Matériaux \& Techniques, 2004, 92 (7-8-9), pp.9 - 15. 10.1051/mattech:2004030 . hal-01744524

\section{HAL Id: hal-01744524 \\ https://hal.sorbonne-universite.fr/hal-01744524}

Submitted on 27 Mar 2018

HAL is a multi-disciplinary open access archive for the deposit and dissemination of scientific research documents, whether they are published or not. The documents may come from teaching and research institutions in France or abroad, or from public or private research centers.
L'archive ouverte pluridisciplinaire HAL, est destinée au dépôt et à la diffusion de documents scientifiques de niveau recherche, publiés ou non, émanant des établissements d'enseignement et de recherche français ou étrangers, des laboratoires publics ou privés. 


\title{
Modifications superficielles de films de dioxyde d'étain créés sous forte polarisation anodique et au potentiel d'abandon en milieux chlorurés
}

\author{
H. Cachet ${ }^{1}$, G. Folcher ${ }^{1}$, S. Haskouri ${ }^{1}$, B. Tribollet $^{1}$ et D. Festy ${ }^{2}$ \\ 1 CNRS-UPR 15, Interfaces et Systèmes Electrochimiques, Université P. et M. Curie, Paris \\ 2 IFREMER, Centre de Brest, Service Matériaux et structures, BP 70, 29280 Plouzané
}

\begin{abstract}
SURFACE MODIFICATIONS OF TIN DIOXIDE FILMS INDUCED UNDER STRONG ANODIC POLARISATION AND AT REST POTENTIAL IN CHLORIDE MEDIA
\end{abstract}

ABSTRACT: Underwater optical instrumentation can be efficiently protected against biofouling by seawater electrolysis on a conductive and transparent tin dioxide coating, without environmental damage. When applying anodic polarisation, biofouling active prevention by free chlorine production was proved to be effective over 5 months at least. Remarkably, a persistent protection effect (passive mode) was observed after switching off the polarisation during a few days. This unexpected effect is advantageous for electrical energy saving. The origin of this effect is not fully understood yet. Preliminary results are presented here on the possible tin dioxide surface modifications undergone during on/off anodic polarisation cycles. On the basis of RHEED observations and EDX analysis, neither new crystallised phase, nor significant amount of chlorine atoms were found under anodic polarisation. But, during anodic/rest potential cycles, it was shown by impedance spectroscopy that a film would be formed during the sequences at rest potential. For comparison purpose, it was shown by EDX that a noticeable quantity of chlorine species could be fixed on a tin electrode electrochemically. From these findings, it is suggested that some reduced tin species might be formed at rest potential, able to react with chloride ions.

RÉSUMÉ: La production localisée de chlore libre par électrolyse contrôlée de l'eau de mer sur un film de dioxyde d'étain constitue un moyen actif de protection d'équipements optiques sousmarins vis-à-vis des biosalissures et sauvegardant l'environnement. La protection sous polarisation anodique s'avère effective sur plusieurs mois. Le fait remarquable est qu'après la coupure de la polarisation, on observe une persistance de l'effet protecteur (mode passif) pouvant atteindre plusieurs jours. Ce constat est mis à profit pour une gestion économe de l'énergie électrique nécessitée par le mode de protection active. En vue de comprendre les origines de ce phénomène, on présente ici les premiers résultats sur les modifications de surface subies par le film de dioxyde d'étain lors de cycles sous/hors polarisation. Sur la base d'observations RHEED et d'analyses EDX, on montre qu'il n'y a, sous polarisation anodique, ni formation d'une phase nouvelle cristallisée, ni fixation de chlore en quantité appréciable. Par contre, lors de cycles polarisation anodique/ potentiel d'abandon, une étude par spectroscopie d'impédance révèle une modification importante des paramètres électriques de l'interface, suggérant la formation d'un film lorsque le système est au potentiel d'abandon. A titre de comparaison, il est montré par EDX qu'une électrode d'étain pouvait fixer une quantité significative de chlore sous certaines conditions de polarisation. Ces résultats suggèrent la formation de sites $\mathrm{Sn}^{2+}$ et/ou $\mathrm{Sn}^{\circ}$, pouvant réagir avec les ions chlorure.

Conférence présentée au Forum "Biodétérioration des matériaux" organisé à La Rochelle les 23 et 24 octobre 2003 par la Commission Biodétérioration des Matériaux du Centre Français de l'Anticorrosion (CEFRACOR). 


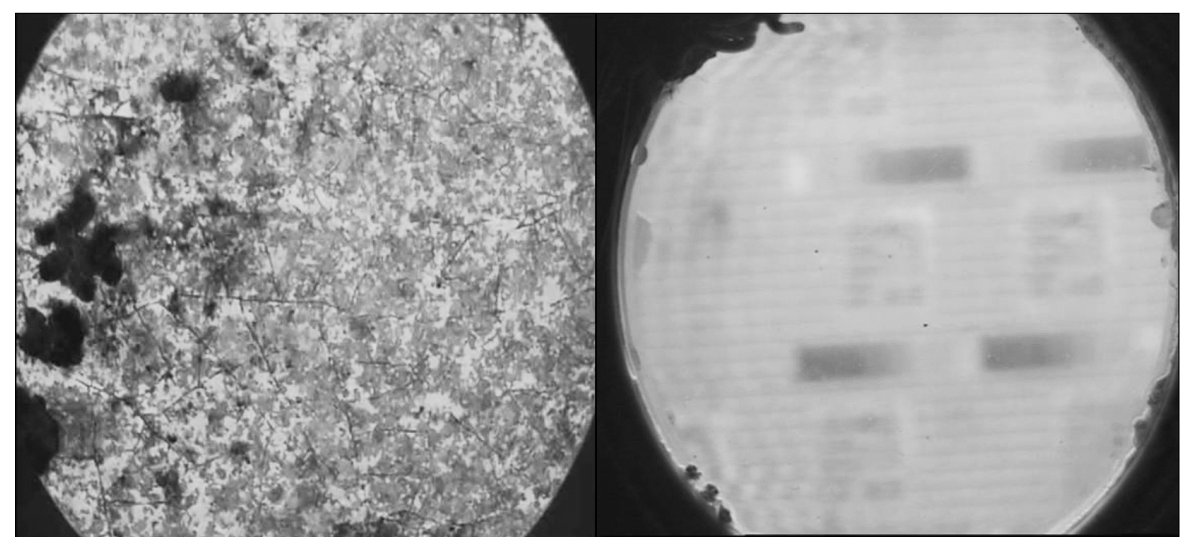

Fig. 1 - Comparaison d'un hublot non protégé (à gauche) et d'un hublot protégé électrochimiquement (à $+1,5 \mathrm{~V} / \mathrm{ECS})$ après immersion en eau de mer naturelle pendant 5 mois.

Fig. 1 - Comparison of an unprotected optical glass window (left) and an electrochemically protected one $(a t+1.5 \mathrm{~V} / \mathrm{SCE})$ after 5 month seawater exposure at 10 meter depth in the Brest bay. couvrant le domaine de concentration de l'eau de mer $[4,5]$. Ces éléments nous ont conduits à (i) retenir une structure à double couche $\mathrm{SnO}_{2}: \mathrm{F} / \mathrm{SnO}_{2}: \mathrm{Sb}(2 \%)$ du film de dioxyde d'étain [1], (ii) fixer des conditions de fonctionnement à potentiel constant, égal à $+1,5 \mathrm{~V} / \mathrm{ECS}[1]$. Comme nous l'avons montré, le mécanisme de corrosion à $\mathrm{pH} 8$ met en jeu la capture d'électrons des liaisons $\mathrm{Sn}-\mathrm{O}$ par des espèces radicalaires $\mathrm{OH}^{\bullet}$ et $\mathrm{Cl}^{\bullet}$ électrogénérées conduisant à la formation de groupements $\mathrm{Sn}-\mathrm{OH}$ et $\mathrm{Sn}-\mathrm{Cl}$ en surface. Une des questions posées est de savoir si une telle chloration de la surface peut ou non constituer tout ou partie du principe actif antibiofilm.

Des travaux archéologiques publiés récemment, portant sur l'état d'objets en étain caractéristiques physicochimiques ont été optimisées en vue de son fonctionnement en tant qu'anode, à des potentiels suffisamment positifs pour produire du chlore actif. L'action biocide est basée essentiellement sur la génération électrochimique d'acide hypochloreux ( $\mathrm{HClO}$ : eau de javel) capable d'empêcher l'accrochage des bactéries et inhibant ainsi la formation d'un biofilm. La description du procédé et sa mise en œuvre sur différents sites ont été présentées antérieurement [1-3]. Le mode de génération par électrochimie à très faible densité de courant présente l'avantage de limiter strictement l'action biocide à la surface même de la fenêtre à protéger, sans action néfaste sur le proche environnement. L'efficacité du procédé de prévention électrochimique du biofilm est illustrée par la figure 1. A ce stade, il permet d'assurer efficacement la protection de hublots immergés en milieu marin vis-à-vis des bio salissures, et équipant divers dispositifs optiques (caméras de surveillance, capteurs...).

Suivant le principe de base de notre procédé, l'action biocide repose sur la génération de chlore actif à la surface du hublot. Toutefois, des observations effectuées à L'IFREMER lors d'essais de longue durée, ont montré qu'une protection persiste sur plusieurs jours en l'absence de polarisation électrique. La compréhension du mécanisme d'action de ce mode de protection passif est un point important, car il permettrait d'envisager la préparation en laboratoire de surfaces modifiées ayant des propriétés antifouling.

Comme première approche, on se propose de vérifier si du " chlore", sous une forme à préciser, serait incorporé dans les couches superficielles du film $\mathrm{SnO}_{2}$ pendant le traitement électrochimique. Ceci inclut non seulement le maintien sous polarisation anodique avec dégagement de $\mathrm{HClO}$ (à +1,5 V/ECS) mais aussi la séquence sans polarisation pendant laquelle la surface voit un potentiel d'abandon voisin de $0 \mathrm{~V} / \mathrm{ECS}$. Ce fort déplacement en potentiel du point de fonctionnement pourrait, d'un point de vue électrochimique, être à l'origine de modifications notables de la surface des films de dioxyde d'étain.

Dans des travaux antérieurs portant sur la stabilité sous polarisation anodique de films $\mathrm{SnO}_{2}$ au contact de solutions de $\mathrm{NaCl}$ à $\mathrm{pH} 8$, nous avons montré que la production de chlore actif s'accompagne d'un phénomène de corrosion dont la vitesse est (i) dépendante du potentiel entre +1 et $+1,4 \mathrm{~V} / \mathrm{ECS}$ avec un maximum à $+1,2 \mathrm{~V} / \mathrm{ECS}$, (ii) d'autant plus faible que la concentration en chlorure est élevée, cette dernière propriété trouvés dans des épaves de navires [6], ont mis en évidence la présence sur ces objets de romarchite $(\mathrm{SnO})$, d'hydroromarchite $\left(5 \mathrm{SnO}-2 \mathrm{H}_{2} \mathrm{O}\right)$ et surtout d'un hydroxochlorure d'étain de formule $\mathrm{Sn}_{3} \mathrm{O}(\mathrm{OH})_{2} \mathrm{Cl}_{2}$ ("rouille" de l'étain ou Abhurite [7]). Le point remarquable est que ces objets recouverts partiellement par ces composés sont totalement exempts de bio-salissures, ce qui d'évidence constitue un élément de réflexion par rapport au problème du caractère antifouling acquis par nos surfaces $\mathrm{SnO}_{2}$ après traitement électrochimique.

Dans le présent travail, on s'est proposé de caractériser les modifications de la surface de films de dioxyde d'étain après des séquences de polarisation/non polarisation dans divers milieux chlorurés artificiels, à différents $\mathrm{pH}$, ainsi qu'en eau de mer naturelle (EMN). Compte tenu des travaux en archéologie sous-marine sur la conservation des objets en étain, on s'est intéressé, à titre de comparaison, au comportement électrochimique d'une électrode d'étain au contact de solutions contenant des ions chlorure.

Les techniques expérimentales utilisées ont été soit des techniques d'analyse ou d'observation ex-situ (diffraction d'électrons par réflexion (RHEED), diffraction de rayons $X$ (XRD), microscopie électronique à balayage et analyse $\mathrm{X}$ en énergie $(\mathrm{MEB}+\mathrm{EDX}))$, soit des techniques électrochimiques in situ (voltampérométrie et spectroscopie d'impédance (SIE)).

\section{Etude par RHEED de la surface de films $\mathrm{SnO}_{2}$ polarisés anodiquement}

On s'est proposé de caractériser toute modification de la surface du dioxyde d'étain par micro diffraction d'électrons (RHEED). Pour cette étude, tous les échantillons ont été préparés sur substrat de silicium afin d'avoir la rugosité minimale pour pouvoir appliquer valablement la technique RHEED. La limite de cette approche est que la technique RHEED ne peut apprécier que les composés cristallisés, ce qui n'est pas obligatoirement le cas des modifications apportées par les maintiens électrochimiques réalisés sous polarisation. Les paramètres expérimentaux ont été le potentiel appliqué $(+1,5 \mathrm{~V} / \mathrm{ECS})$, la nature de l'électrolyte aqueux (chlorure, perchlorate, sulfate) à $\mathrm{pH} 8$, le temps d'immersion.

La référence de l'étude est le diagramme RHEED d'un échantillon non modifié (fig.2a). Compte tenu de la nature polycris- 

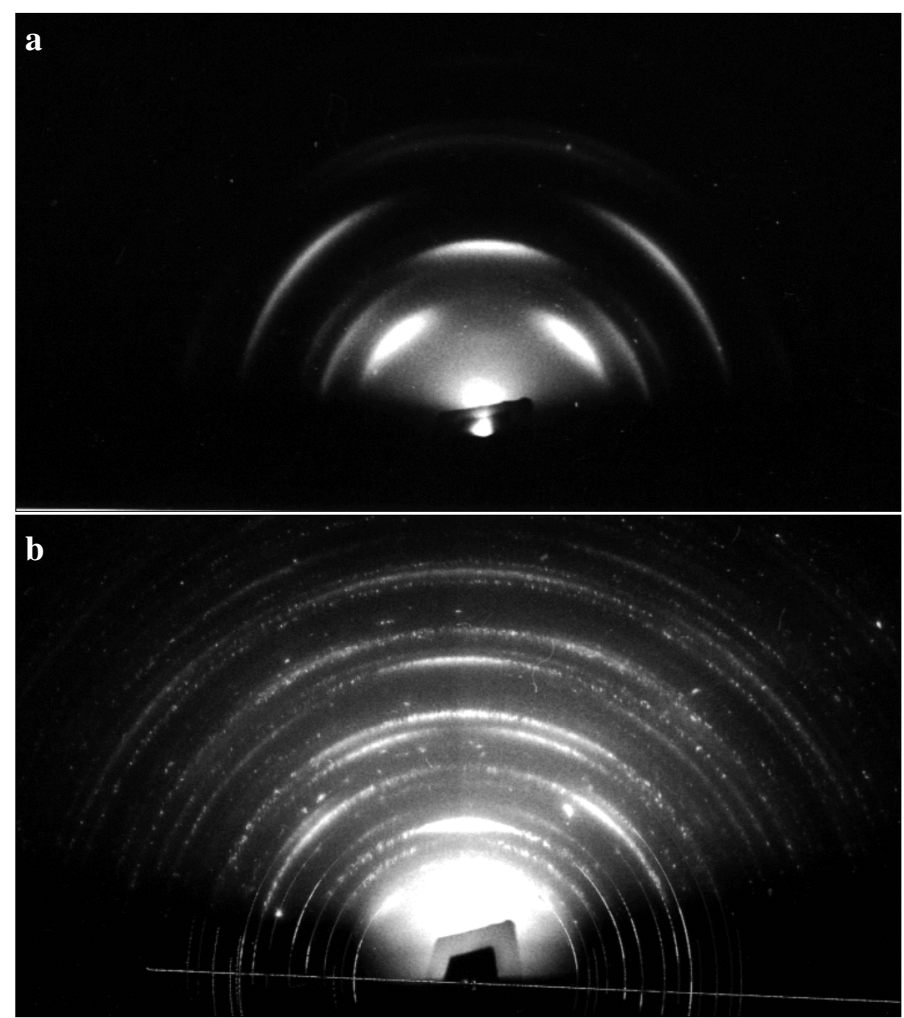

Fig. 2 - Diagrammes RHEED de films SnO, déposés sur substrat de silicium : (a) film non utilisé; (b) film ayant été polarisé anodiquement à $+1,5 \mathrm{~V} / \mathrm{ECS}$.

Fig. 2 - RHEED diagrams of $\mathrm{SnO}_{2}$ films deposited on silicon substrates: (a) as prepared, unused film; (b) film polarised at $+1.5 \mathrm{~V} / \mathrm{SCE}$.

talline de nos films, le diagramme RHEED est caractérisé par une succession d'anneaux concentriques dont la position et les intensités relatives sont en accord avec la structure cassitérite tétragonale ( $a=0.4730 \mathrm{~nm} ; c=0.3188 \mathrm{~nm})$, en accord avec la fiche ASTM n ${ }^{\circ}$ 21-1250. Les films $\mathrm{SnO}_{2}$ déposés par pyrolyse de spray sont fortement texturés avec une orientation préférentielle $<100>$. La figure $2 b$ est un exemple typique de ce que l'on obtient après un maintien électrochimique dans le domaine fortement anodique. Le diagramme est effectivement modifié, mais il n'y a pas d'évidence d'un nouveau composé de type hydroxochlorure d'étain (fiches ASTM N ${ }^{\circ}$ 39-314 pour le composé de formule $\mathrm{Sn}_{3} \mathrm{O}(\mathrm{OH})_{2} \mathrm{Cl}_{2}$, ASTM N $\mathrm{N}^{\circ}$ 15-675 pour le composé de formule $\left.\mathrm{Sn}_{4}(\mathrm{OH})_{6} \mathrm{Cl}_{6}\right)$. La modification du diagramme est essentiellement liée à l'effet de la corrosion en milieu chlorure qui induit une perte de la texture du film à l'extrême surface. On fait ainsi réapparaître des anneaux de diffraction du $\mathrm{SnO}_{2}$ qui étaient masqués par l'intensité des figures de diffraction dues à la texture initiale.

En complément, on a vérifié si les propriétés de mouillage de la surface de $\mathrm{SnO}_{2}$ étaient modifiées après polarisation anodique. La figure 3 montre clairement le changement de caractère de la surface de l'oxyde suivant qu'elle a été polarisée anodiquement ou non. Initialement, la surface est hydrophile, la goutte d'eau s'étalant très largement sur la surface (partie rectangulaire de l'échantillon). Après polarisation d'une partie de l'échantillon (extrémité “ pointue"), on constate qu'une goutte d'eau conserve une forme quasi-sphérique. L'acquisition d'un caractère hydrophobe est certes favorable à une moins bonne adhérence du biofilm mais il n'est pas en soi suffisant pour expliquer l'effet rémanent antisalissure.

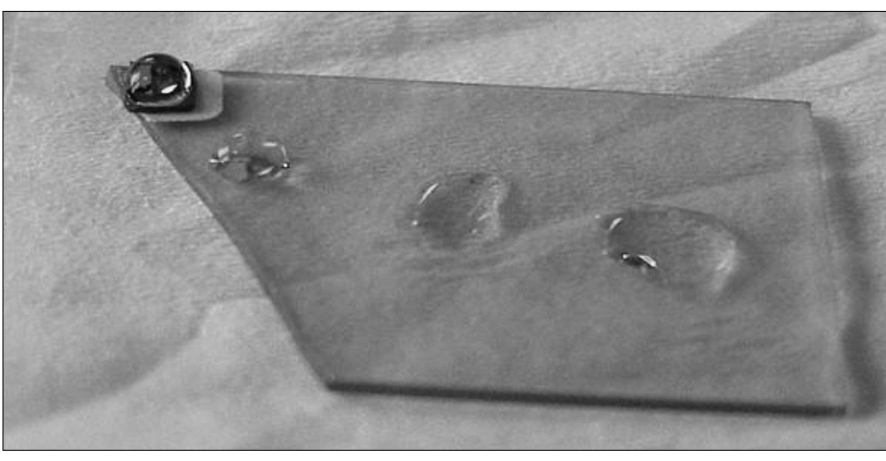

Fig. 3 - Modification des propriétés de mouillage après fonctionnement sous polarisation anodique en milieu chlorure, l'état initial étant représenté par la partie droite de l'échantillon.

Fig. 3 - Modification of wetting properties of a $\mathrm{SnO}_{2}$ film after anodic polarisation in a $0.5 \mathrm{M}$ chloride solution (hydrophilic initial state depicted by the right part of the sample).

Ainsi, la polarisation anodique en milieu chloruré ne conduit pas à la formation d'un composé cristallisé mais induit (i) une perte de texture, c'est-à-dire un certain désordre à la surface, corrélé avec une augmentation de la rugosité, (ii) l'acquisition d'un caractère hydrophobe.

\section{Etude par EDX de la surface de films $\mathrm{SnO}_{2}$ polarisés anodiquement}

La figure 4a montre le spectre EDX d'une électrode verre/ $\mathrm{SnO}_{2}: \mathrm{F} / \mathrm{SnO}_{2}: \mathrm{Sb}$ ayant fonctionné sous protection anodique à $+1,5 \mathrm{~V} / \mathrm{ECS}$ pendant 26 heures dans une solution $\mathrm{KCl} 0,5 \mathrm{M}$ à $\mathrm{pH}$ 10. Comme le montre l'agrandissement de la région du pic

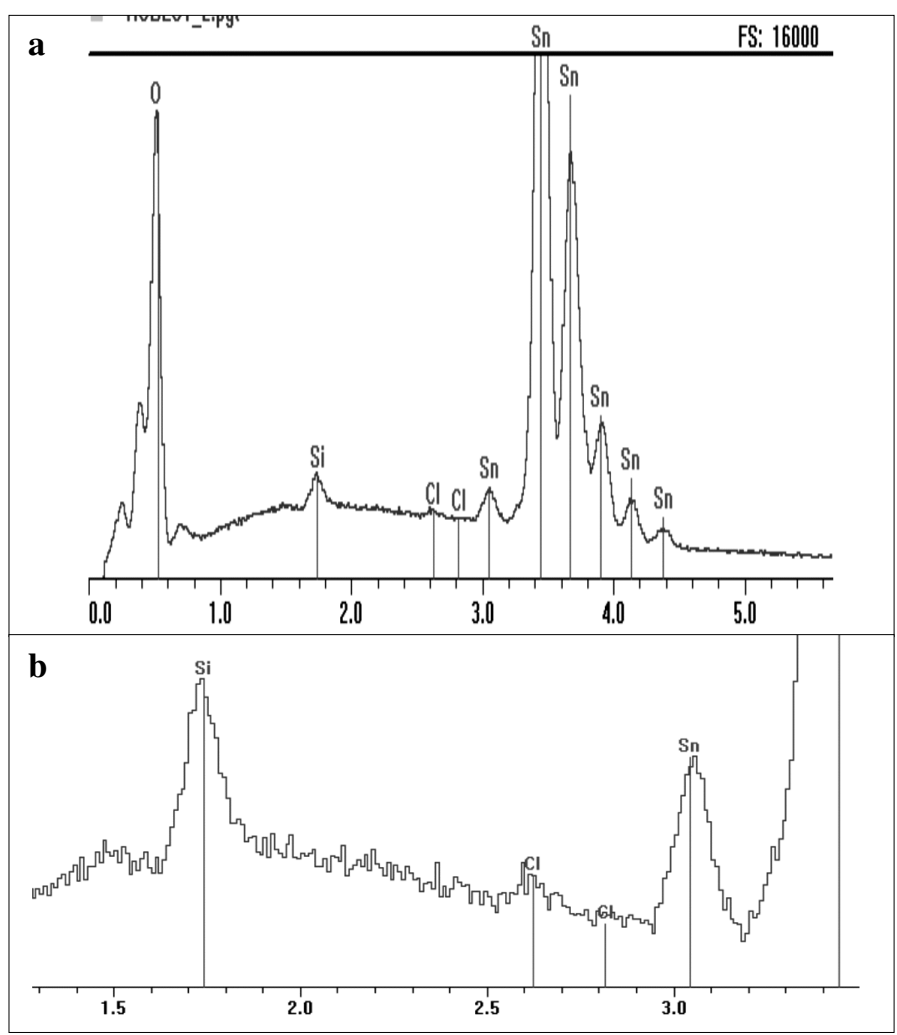

Fig. 4 - (a) Spectre EDX d'une électrode ayant fonctionné sous protection anodique en milieu KCl 0,5M à pH 10 pendant $26 \mathrm{~h}$; (b) zoom sur le pic Cl. Fig. 4 - (a) EDX spectrum of a $\mathrm{SnO}_{2}$ electrode polarised at $+1.5 \mathrm{~V} / \mathrm{SCE}$ in $0.5 \mathrm{M} \mathrm{KCl}$ at $\mathrm{pH} 10$ for 26 hours; (b) zoom on the Cl peak. 


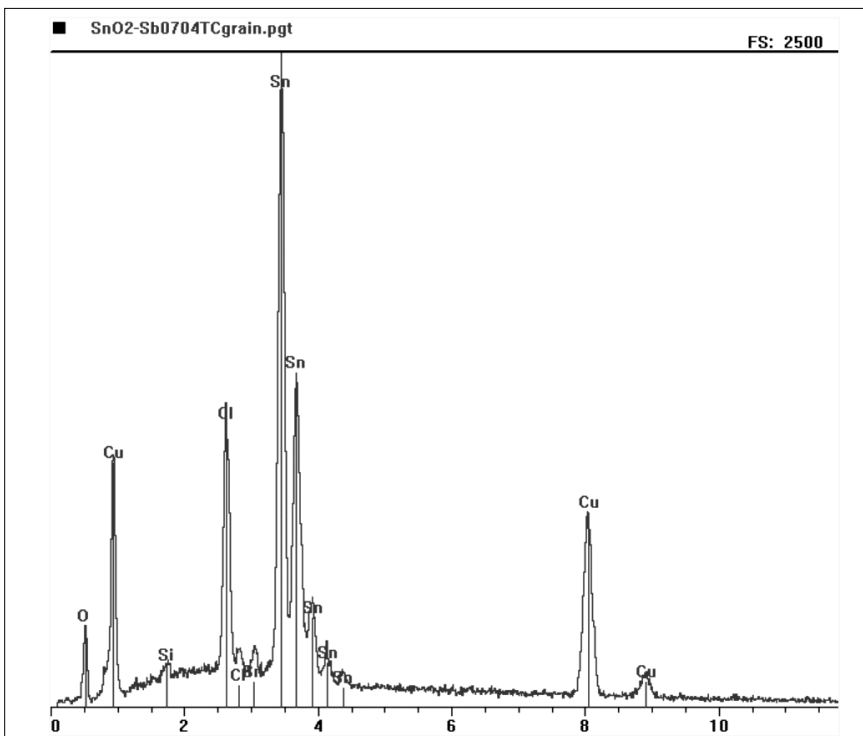

Fig. 5 - Spectre EDX d'une électrode $\mathrm{SnO}_{2}$ immergée dans de l'eau de mer naturelle après une séquence polarisation/non polarisation.

Fig. 5 - EDX spectrum of a $\mathrm{SnO}_{2}$ electrode immersed in seawater, after a on/off polarisation cycle.

"Cl” (fig.4b), la présence de chlore est à peine significative, à la limite du seuil de détection de la méthode. La fraction atomique en $\mathrm{Cl}$ par rapport aux éléments $\mathrm{Sn}, \mathrm{Si}, \mathrm{O}$ et $\mathrm{Cl}$ est de $\approx 0,1 \%$. Le spectre étant similaire à celui d'un film n'ayant jamais travaillé, on peut penser que le chlore vient simplement de l'incorporation involontaire d'atomes de chlore lors de la préparation sachant que le précurseur d'étain utilisé est $\mathrm{SnCl}_{4}$ [8]. Le fonctionnement en régime anodique ne semble donc pas apporter un enrichissement significatif en chlore.

Des analyses EDX ont été entreprises sur des électrodes $\mathrm{SnO}_{2}$ au contact d'eau de mer naturelle. Une des électrodes n'a subi aucune mise sous polarisation et a été simplement immergée environ 48 heures. La seconde a été polarisée à 1,5 V/ECS pendant 48 heures, puis laissée au potentiel d'abandon pendant 24 heures. Les résultats EDX montrent que la quantité de chlore fixée à la surface de l'électrode $\mathrm{SnO}_{2}$ est importante après la séquence polarisation/non polarisation (fig. 5), par comparaison avec le cas de l'électrode non traitée (fig. 6).

\section{Comportement électrochimique d'une électrode d'étain en milieu chloruré}

Les études archéologiques en milieu marin portant sur des objets en étain immergés deux à trois cents ans ont mis en évidence la transformation superficielle de l'étain soit en oxyde non stoechiométrique $\mathrm{SnO}_{x}$, soit de manière prépondérante en hydroxochlorure d'étain, de formule $\mathrm{Sn}_{3} \mathrm{O}(\mathrm{OH})_{2} \mathrm{Cl}_{2}$ [6]. La présence de ce composé empêche efficacement le biofilm de se développer sur les objets en étain métallique. Il n'y a actuellement aucune information sur la cinétique de formation de ce composé. Si le caractère antifouling semble bien effectif, il faut souligner que ce composé se développe à partir de l'étain métal Sn, c'est-à-dire au degré d'oxydation zéro, et que la situation est différente dans le cas de $\mathrm{SnO}_{2}$ pour lequel l'étain est au degré d'oxydation (+4). Ces données sur l'évolution de l'étain en milieu marin nous ont amenés à entreprendre des expériences sur le comportement électrochimique de l'étain en

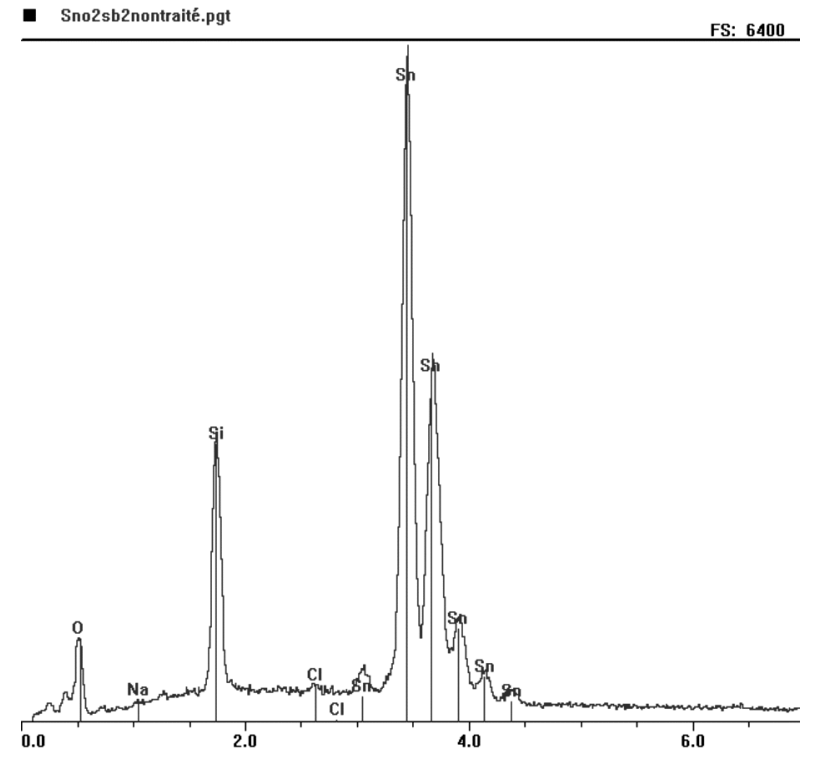

Fig. 6 - Spectre EDX d'une électrode $\mathrm{SnO}_{2}$ immergée dans de l'eau de mer naturelle non traitée électrochimiquement.

Fig. 6 - EDX spectrum of a SnO2 electrode simply immersed in seawater, without any electrochemical treatment.

régime d'oxydation des ions chlorure à différents $\mathrm{pH}$ basiques. Notons que sur l'étain, le dégagement du chlore a lieu à des potentiels nettement moins positifs (environ $+0,8 \mathrm{~V} / \mathrm{ECS}$ ) que pour $\mathrm{SnO}_{2}$.

Une étude systématique par EDX a été entreprise sur des surfaces d'étain immergées dans des solutions tampon à $\mathrm{pH} 8,10$

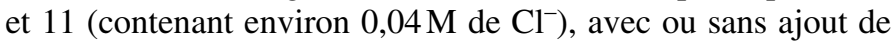
0,2 ou $0,5 \mathrm{M}$ d'ions chlorure, puis soumises à plusieurs balayages en potentiel à $10 \mathrm{mV} \cdot \mathrm{s}^{-1}$ entre deux bornes $\mathrm{E}_{1}$ et $\mathrm{E}_{2}$. Le tableau I regroupe les systèmes étudiés, les conditions expérimentales, ainsi que la quantité de chlore incorporée en prenant comme estimateur le rapport de l'intensité du pic $\mathrm{Cl}$ à 2,62 keV à celle du pic Sn situé à 3,45 keV.

Des résultats rassemblés dans le tableau I, il ressort que la quantité de chlore fixée sur l'électrode d'étain est d'autant plus grande que la concentration en $\mathrm{Cl}^{-}$dans l'électrolyte est élevée

Tableau I - Etude par EDX de la fixation du chlore sur des électrodes d'étain suivant le pH, la concentration en ions chlorure, le domaine de potentiel.

Table I - EDX study of chlorine fixation on tin electrodes as a function of $\mathrm{pH}$, chloride ions concentration, voltage range.

\begin{tabular}{|c|c|c|c|c|c|}
\hline Système & $\mathbf{p H}$ & $\begin{array}{c}\text { [Cl- }] \\
\mathbf{M}\end{array}$ & $\begin{array}{c}\mathbf{E}_{\mathbf{1}} \\
\text { V/ECS }\end{array}$ & $\begin{array}{c}\mathbf{E}_{\mathbf{2}} \\
\text { V/ECS }\end{array}$ & $\begin{array}{c}\mathbf{I}(\mathbf{C l}) / \mathbf{I}(\mathbf{S n}) \\
\%\end{array}$ \\
\hline Tampon borate & 8 & 0,04 & $-0,8$ & $-0,3$ & 0 \\
\hline Tampon + KCl & 8 & 0,2 & $-0,8$ & $-0,3$ & 6 \\
\hline Tampon + KCl & 8 & 0,5 & $-0,7$ & $-0,4$ & 21 \\
\hline $\begin{array}{c}\text { Tampon/immersion } \\
\text { simple }\end{array}$ & 8 & 0,04 & néant & néant & 6 \\
\hline Tampon + KCl & 10 & 0,5 & $-0,7$ & $+1,5$ & 0 \\
\hline Tampon + NaCl & 10 & 0,5 & $-0,8$ & $-0,3$ & 20 \\
\hline Tampon & 11 & 0,04 & $-0,7$ & $+0,9$ & 4 \\
\hline Tampon & 11 & 0,04 & $-0,8$ & $-0,3$ & 9 \\
puis $-1,5$ & $+1,5$ & 6 \\
\hline Tampon + KCl & 11 & 0,5 & $-0,8$ & $-0,3$ & 6 \\
\hline Poudre (Sn,O,Cl) & 11 & & & & $15-20$ \\
\hline
\end{tabular}




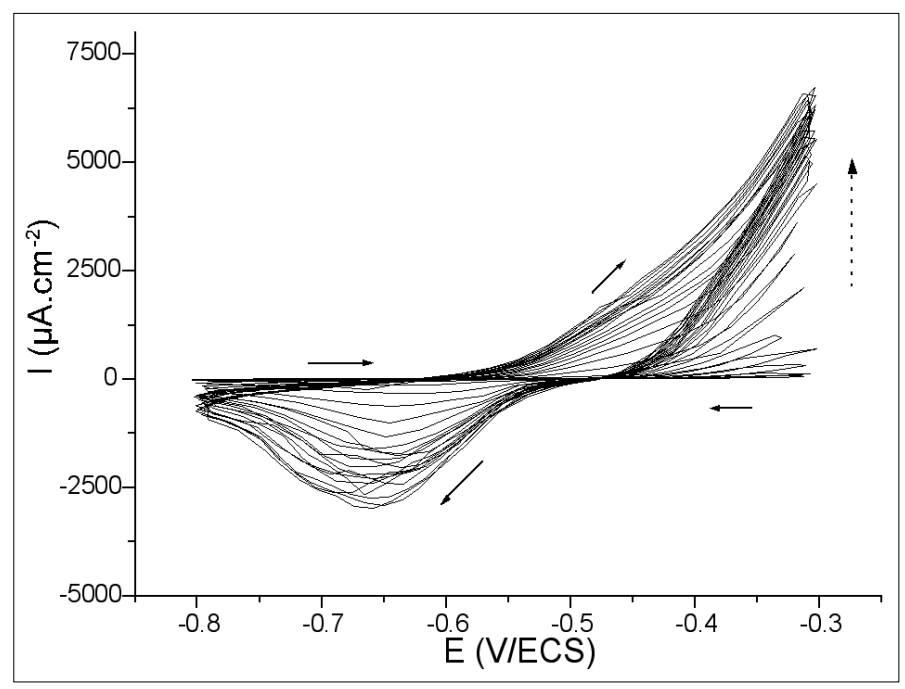

Fig. 7 - Voltamétrie cyclique pour une électrode d'étain au contact d'une solution $0,5 \mathrm{M} \mathrm{KCl}$ à $\mathbf{p H ~ 8}$, à la vitesse de balayage de $20 \mathrm{mV} \cdot \mathrm{s}^{-1}$.

Fig. 7 - Cyclic voltamperometry of a tin electrode in a $0.5 \mathrm{M} \mathrm{KCl}$ solution at $\mathrm{pH}$ 8 ; potential scan rate : $20 \mathrm{mV} . \mathrm{s}^{-1}$.

et que l'électrode ne "voit" pas de potentiels trop positifs. Un taux d'incorporation de $20 \%$ a été obtenu en solution $\mathrm{KCl}$ $0,5 \mathrm{M}$ à $\mathrm{pH} 8$ en limitant strictement le domaine de potentiel à la région cathodique. Ce taux est tout à fait comparable à celui observé pour une poudre $(\mathrm{Sn}, \mathrm{Cl}, \mathrm{O})$ que nous avons obtenue à partir de la corrosion de l'étain métallique en milieu chlorure à pH 11 (voir ci-après). La figure 7 montre l'allure de la courbe courant-tension d'une électrode d'étain au contact d'une solution $\mathrm{KCl} 0,5 \mathrm{M}$ à $\mathrm{pH} 8$, polarisée entre $-0,8$ et $-0,3 \mathrm{~V} / \mathrm{ECS}$ à la vitesse de $20 \mathrm{mV} . \mathrm{s}^{-1}$. Après quelques cycles, on observe une activation notable de l'électrode suivie par la formation d'une poudre blanchâtre dont une partie est soluble, une autre partie est déposée sur l'électrode en même temps qu'un dépôt noirâtre d'oxyde d'étain $\mathrm{SnO}_{\mathrm{x}}$. La réponse voltampérométrique est en accord avec la réaction redox suivante:

$$
\mathrm{Sn}(\mathrm{OH}) \mathrm{Cl} \cdot \mathrm{H}_{2} \mathrm{O}+2 \mathrm{e}^{-} \Leftrightarrow \mathrm{Sn}+\mathrm{OH}^{-}+\mathrm{Cl}^{-}+2 \mathrm{H}_{2} \mathrm{O}
$$

dont le potentiel standard est $-0,60 \mathrm{~V} / \mathrm{ECS}$ à $\mathrm{pH}$ 8, d'après [9]. Par contre, à $\mathrm{pH} 8$, sans ajout de $\mathrm{KCl}$, la courbe de voltamétrie cyclique montre une passivation progressive de l'électrode avec la formation vraisemblable de $\mathrm{Sn}(\mathrm{OH})_{2}$. Il n'y a pas formation d'un dépôt blanchâtre dans le milieu tampon seul. A pH 11, dans les mêmes conditions, la solution devient blanchâtre, indiquant la formation d'espèces colloïdales que l'on a pu récupérer par simple filtration sur papier. Comme ci-dessus, l'analyse EDX révèle aussi la présence de chlore sur l'électrode. L'analyse EDX de la poudre récupérée indique la présence des éléments $\mathrm{Sn}, \mathrm{Cl}$ et $\mathrm{O}$. Toutefois le diagramme de diffraction $\mathrm{X}$ de cette poudre ne correspond pas à celui de l'abhurite. Il s'agirait donc d'une autre variété d'hydroxochlorure d'étain. La différence de comportement entre $\mathrm{pH} 8$ et $\mathrm{pH} 11$ peut raisonnablement être reliée au changement d'espèce dominante en fonction du $\mathrm{pH}$ [10]. A $\mathrm{pH} 8$, l'espèce oxydée dominante est $\mathrm{Sn}(\mathrm{OH})_{2}$, alors qu'à $\mathrm{pH} 11$ il s'agit d'une espèce ionique $\mathrm{Sn}(\mathrm{OH})_{3}{ }^{-}$, les deux espèces impliquant $\mathrm{Sn}(\mathrm{II})$. Du fait de la stabilité de $\mathrm{SnO}_{2}$, il n'existe pratiquement aucun travail sur la complexion de $\mathrm{Sn}(\mathrm{IV})$ avec $\mathrm{Cl}^{-}$. Dans le cas de $\mathrm{Sn}(\mathrm{II})$, il peut y avoir complexation avec $\mathrm{Cl}^{-}$mais plutôt à des $\mathrm{pH}$ acides, inférieurs à 4. De ces travaux sur la corrosion de l'étain métallique en milieu chlorure concentré ( $>0,2 \mathrm{M}$ ), il ressort que quel que soit le $\mathrm{pH}$ entre 8 et 11 , il y a fixation de chlore à la surface d'une électrode d'étain, tant que l'électrode ne subit pas de fortes polarisations anodiques.

\section{Effet des cycles sous/hors polarisation suivi par spectroscopie d'impédance}

Afin de regarder l'effet des cycles de polarisation/non polarisation, nous avons cherché à suivre l'évolution de paramètres électriques caractéristiques de l'interface électrochimique et pouvant donner des informations nouvelles sur les modifications de surface subies par l'électrode $\mathrm{SnO}_{2}$. En vue d'utiliser la spectroscopie d'impédances électrochimiques, nous avons défini le protocole suivant: une électrode $\mathrm{SnO}_{2}$ est maintenue à $+1,5 \mathrm{~V} / \mathrm{ECS}$ pendant 48 heures en eau de mer naturelle. Le courant et un spectre d'impédance entre $60 \mathrm{kHz}$ et $0,001 \mathrm{~Hz}$ sont enregistrés toutes les 2 heures (Solartron 1287/1255 et logiciel FRACOM $^{\mathrm{TM}}$ ). La polarisation est interrompue pendant une période de 1 ou 2 jours. Le cycle avec/sans polarisation est repris alternativement. On peut suivre ainsi l'évolution de la résistance série et de la capacité interfaciale en fonction du temps et du cyclage.

Les diagrammes d'impédance enregistrés sous une polarisation anodique de $+1,5 \mathrm{~V} / \mathrm{ECS}$ présentent une boucle principale centrée vers quelques dizaines de $\mathrm{Hz}$, que nous appellerons boucle MF (pour moyennes fréquences). Le comportement à plus basses fréquences est plus complexe. Il tend vers un comportement de type diffusionnel, en relation vraisemblablement avec la réaction d'oxydation des ions chlorure. La boucle MF est bien définie. Toutefois, les agrandissements de la partie HF (pour hautes fréquences) révèlent la présence d'un épaulement plus ou moins visible. Ce dernier peut suggérer la présence d'une couche diélectrique à la surface de l'électrode $\mathrm{SnO}_{2}$. Après un séjour au potentiel d'abandon, et juste après remise sous polarisation anodique à $+1,5 \mathrm{~V} / \mathrm{ECS}$, cette contribution est nettement visible. Par contre, elle tend à disparaître au cours du temps sous polarisation. Ce résultat suggère que la couche se formant au potentiel d'abandon ne serait pas stable sous forte polarisation anodique.

Une exploitation simplifiée des spectres d'impédance, essentiellement à partir de la boucle MF, a permis de visualiser les variations de la capacité interfaciale $C_{d}$ (fig. 8) et de la résistance série $R_{s}$ (fig. 10). Ces variations sont à comparer à celles du

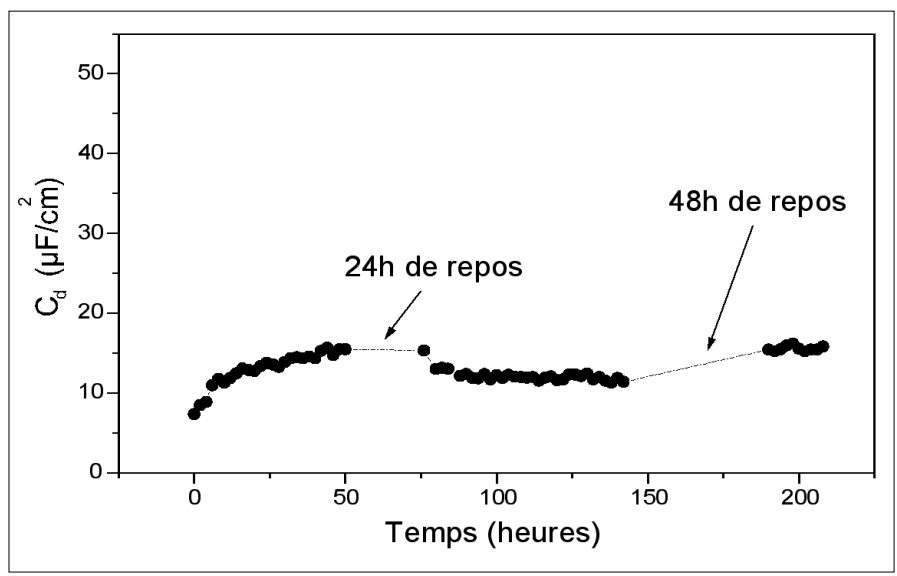

Fig. 8 - Evolution de la capacité interfaciale mesurée à + 1,5 V/ECS au cours de cycles sous/hors polarisation anodique.

Fig. 8 - Variations of the interfacial capacitance determined at $+1,5 \mathrm{~V} / \mathrm{ECS}$ during on/off anodic polarisation cycles. 


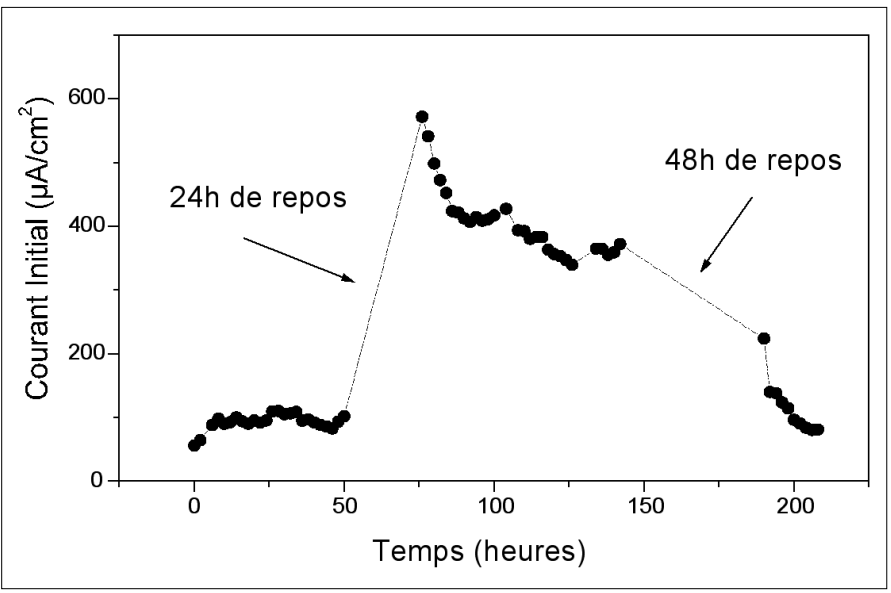

Fig. 9 - Evolution de la densité de courant à + 1,5 V/ECS au cours de cycles sous/hors polarisation anodique.

Fig. 9 - Variations of the current density determined at $+1,5$ V/ECS during on/off anodic polarisation cycles.

courant continu enregistré en même temps que les données d'impédance (fig. 9). La capacité interfaciale augmente régulièrement pendant la première séquence sous polarisation anodique (fig. 8). Cela peut traduire une augmentation de la rugosité résultant du phénomène de corrosion, certes faible, mais coexistant avec la réaction d'oxydation des ions chlorure. La valeur de $C_{d}$ varie de 8 à environ $15 \mu \mathrm{F} / \mathrm{cm}^{2}$. Elle est de toute façon très supérieure à la valeur qui correspondrait à la charge d'espace du film $\mathrm{SnO}_{2}$ sous fort régime d'appauvrissement. On peut donc en conclure que l'électrode $\mathrm{SnO}_{2}$ a perdu son caractère semiconducteur et a acquis un comportement de type métallique. A noter que par la suite cette capacité évolue peu avec le temps ou avec l'alternance des séquences polarisation/ non polarisation.

La figure 9 montre de façon spectaculaire une augmentation du courant après une séquence de 24 heures au potentiel d'abandon. Ceci traduit une forte activation électrochimique de l'électrode, activation qui se réduit progressivement avec le temps de maintien sous polarisation anodique. Après une séquence de 48 heures au potentiel d'abandon, l'activation est moins marquée qu'après seulement 24 heures. On peut en conclure qu'au potentiel d'abandon il se produit une modification de la surface de $\mathrm{SnO}_{2}$ induisant une activation électrochimique de l'électrode, dont la stabilité serait limitée dans le temps.

Après une séquence de polarisation à $+1,5 \mathrm{~V}$, on a mesuré la valeur du potentiel d'abandon en fonction du temps. On constate durant la première heure une forte évolution du potentiel de l'électrode depuis une valeur positive $(+0,3 \mathrm{~V} / \mathrm{ECS})$ à une valeur légèrement négative, vers $-0,2 \mathrm{~V} / \mathrm{ECS}$. Cette valeur

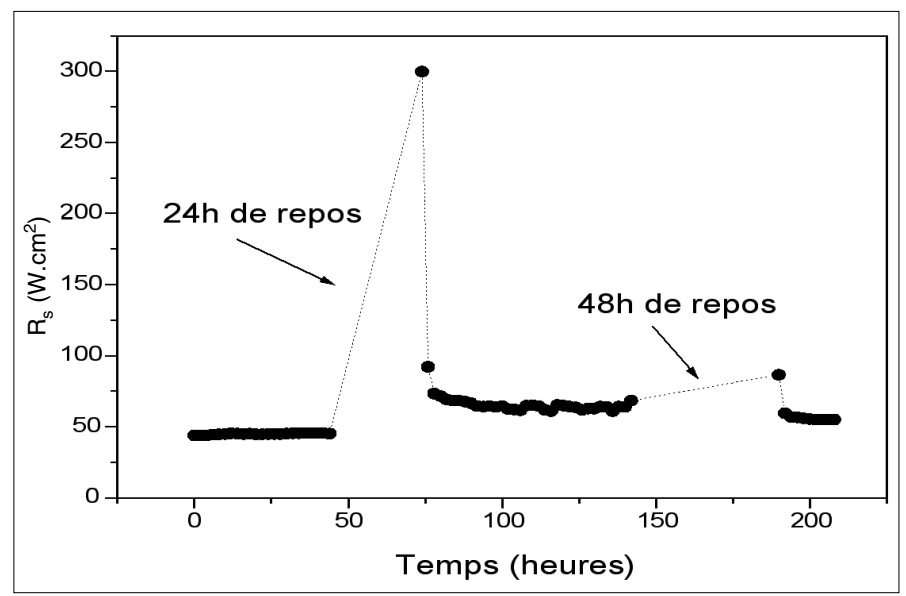

Fig. 10 - Evolution de la résistance série mesurée à $+1,5$ V/ECS au cours de cycles sous/hors polarisation anodique.

Fig. 10 - Variations of the series resistance determined at $+1,5$ V/ECS during on/off anodic polarisation cycles.

est proche des potentiels d'oxydo-réduction des couples $\mathrm{Sn} / \mathrm{Sn}^{2+}$ et $\mathrm{Sn}^{4+} / \mathrm{Sn}^{2+}$. On est donc conduit à penser que l'activation de l'électrode serait liée à la production de sites $\operatorname{Sn}(0)$ et/ou $\mathrm{Sn}^{2+}$. Ces sites peuvent réagir au contact de l'eau de mer comme l'étain métal en fixant des groupements hydroxyde et chlore.

Les variations de la résistance série $R_{e}$ avec l'histoire de l'électrode (fig. 10) mettent en évidence une nette augmentation de $R_{e}$ après un certain temps passé au potentiel d'abandon. Là encore, l'augmentation est beaucoup plus importante après $24 \mathrm{~h}$ qu'après $48 \mathrm{~h}$. $R_{e}$ décroît ensuite très rapidement, puis se stabilise lors du maintien sous polarisation anodique. Ceci est une indication forte de la formation d'une couche au potentiel d'abandon, instable sous polarisation anodique.

Ces résultats sont à rapprocher de ceux obtenus par EDX sur une électrode $\mathrm{SnO}_{2}$ en eau de mer naturelle. Ces derniers montrent que la quantité du chlore fixée à la surface de l'électrode $\mathrm{SnO}_{2}$ est très importante après la séquence polarisation/non polarisation (fig. 5), par comparaison avec l'électrode non traitée (fig. 6). Ces résultats tendent à prouver que lorsque l'électrode est laissée hors polarisation, il y a formation d'une couche amorphe, contenant du chlore, de l'étain sous forme $\mathrm{Sn}^{\circ}$ et $\mathrm{Sn}^{2+}$. Elle n'est pas détectable par les méthodes de diffraction (RHEED). Cette couche n'est pas très stable en fonction du temps au-delà de 24 heures et se détruit sous polarisation anodique. Par analogie avec le comportement de l'étain métal en milieu marin, cette couche pourrait être à l'origine de la protection anti-biosalissure effectivement observée lors des essais sur site.

\section{Conclusions}

L'objectif du présent travail était de tenter d'appréhender le processus responsable de la persistance de l'effet protecteur vis-à-vis de la formation du biofilm. Les études structurales ont montré qu'aucun produit cristallisé nouveau ne se formait sous polarisation anodique, et que les seules modifications résultantes étaient une perte de texture du film $\mathrm{SnO}_{2}$. Schématiquement, l'étape de polarisation anodique est nécessaire pour rendre la surface du film $\mathrm{SnO}_{2}$ plus réactive, mais non suffisante pour lui conférer des propriétés antisalissures. Ce serait donc dans la phase hors polarisation qu'un caractère antifouling serait acquis. Le film $\mathrm{SnO}_{2}$ se trouve alors à des potentiels correspondant aux réactions d'oxydo-réduction de l'étain. Ces conditions électrochimiques peuvent recréer, au moins au niveau de certains sites, une situation comparable à celle d'une électrode d'étain interagissant avec de l'eau de mer. On sait que, dans ce dernier cas, la formation d'hydroxochlorure d'étain devient possible et est capable de protéger des objets en étain des bio salissures. 


\title{
RÉFÉRENCES
}

[1] H. Cachet, D. Festy, G. Folcher, F. Mazeas, B. Tribollet, Matériaux \& Techniques, 7-8 (2002) p. 37.

[2] T. El Moustafid, H. Cachet, B. Tribollet, D. Festy, N. LaCOTTE, Proceedings of the $11^{\text {th }}$ Asian-Pacific Corrosion Control Conference, HoChiMinh City, Vietnam, nov. 1999, Vol. 1, p. 223.

[3] D. Festy, S. Le Bras, M. Clegg, N. Lacotte, M. Lehaitre, R. Menlove, P. Sebastiao, Proceedings of Ocean'98, Nice 1998, France.

[4] H. Cachet, M. Froment, F. Zenia, J. Electrochem. Soc., 143 (1996) p. 442.

[5] H. Cachet, F. Zenia, M. Froment, J. Electrochem. Soc., 146 (1999) p. 977.

[6] Stacie E. DunKLE, Romarchite and other corrosion phases on metal artefacts from the Queen Anne's Revenge (1718). Master of
Science in Geological Sciences, April 2002, Virginia Polytechnic Institute and State University, Blacksburg, Virginia (USA).

[7] Abhurite Mineral data: site Internet webmineral.com/data/ Abhurite.shtml. Données cristallographiques: système trigonaltrapézoïdal; symbole H-M (3 2); groupe d'espace: R32; axes: $a=10,018, c=44,03$, masse volumique: $4.35 \mathrm{~g} . \mathrm{cm}^{-3}$. Données XRD : $d_{h k l}\left(I / I_{0}\right): 2,53(1) 2,8915(0.7) 4,139(0.5)$.

[8] A. Messad, H. Cachet, M. Froment, J. Bruneaux, J. Mater. Sci., 29 (1994) p. 5095

[9] Standard Potentials in Aqueous Solutions, IUPAC, éditeurs A.J. Bard, R. Parsons et J. Jordan, Marcel Dekker Inc., New York (1985).

[10] F. Séby, M. Potin-Gautier, E. Giffaut, O.F.X. Donard, Geochimica \& Cosmochimica Acta, 65 (2001) p. 3041.

\section{La microstructure des aciers et des fontes Genèse et interprétation}

\author{
Madeleine Durand-Charre, Institut National Polytechnique de Grenoble
}

Les 400 pages de cette monographie sont illustrées par de nombreuses micrographies d'alliages commerciaux ou de laboratoire, à toutes les échelles disponibles avec les moyens d'investigation modemes de microscopie électronique, sans oublier la macroscopie/microscopie optique. La première partie du livre est une introduction historique sur l'évolution des structures métallurgiques forgées manuellement depuis la connaissance du fer, en particulier les fameuses structures dites damassées.

Le livre expose en deuxième partie les notions fondamentales de façon à fournir toutes les bases de raisonnement nécessaires sur les équilibres de phases et la cinétique des transformations. Concernant les équilibres de phases, de nombreux diagrammes sont inclus dans leur version la plus récente. La lecture des systèmes temaires est analysée à propos de six systèmes représentatifs des réactions rencontrées dans les aciers: $\mathrm{Fe}-\mathrm{Cr}-\mathrm{C}, \mathrm{Fe}-\mathrm{Ni}-\mathrm{Cr}, \mathrm{Fe}-\mathrm{Mn}-\mathrm{S}, \mathrm{Fe}-\mathrm{Co}-\mathrm{Cu}$, $\mathrm{Fe}-\mathrm{Mo}-\mathrm{Cr}$ et $\mathrm{Fe}-\mathrm{C}-\mathrm{V}$.

Les structures de solidification sont étudiées à travers tous les cas classiques mais aussi dans d'autres moins banals comme les marquages des réactions péritectique ou métatectique ou des cascades de transformations. Les transformations en phase solide sont illustrées et commentées à l'aide des interprétations récentes, en particulier dans le cas des structures bainitiques. De nombreuses références permettent d'approfondir les aspects non développés.

La troisième partie est un guide pour comprendre l'optimisation des aciers, définir la relation entre la microstructure et les propriétés à travers le rôle des éléments d'alliage et celui des divers traitements. Les alliages à base fer sont classés en familles depuis les aciers doux jusqu'aux fontes et aux aciers très alliés.

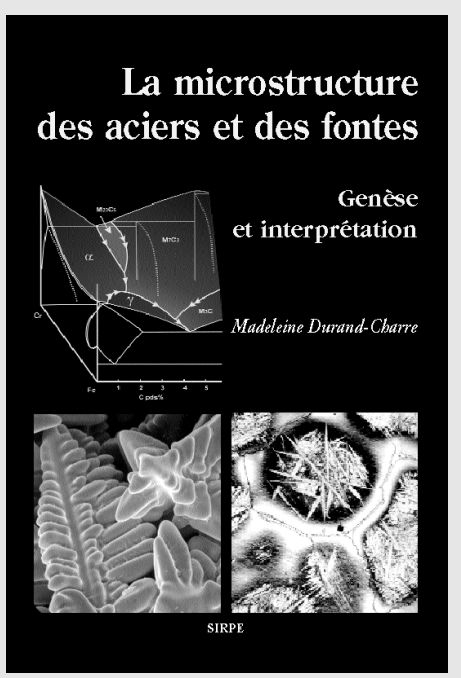

1 Du fer primitif à l'acier du forgeron

2 Les aciers damassés

3 Les phases importantes

dans les aciers

4 Les diagrammes de phases

5 Genèse de la microstructure de solidification

6 Transformation de la microstructure en milieu liquide/solide

\section{TABLE DES MATIERES}

7 Grains, joints de grains et interfaces

8 La diffusion

9 La décomposition de l'austénite

10 La transformation perlitique

11 La transformation martensitique

12 La transformation bainitique

13 La précipitation

14 L'optimisation des nuances d'aciers

15 Macrostructures de solidification
$16 \mathrm{Macro} / \mathrm{microstructures} \mathrm{frittées}$

17 Les aciers peu alliés

18 Les aciers à traitements thermiques

19 Les aciers inoxydables

20 Les aciers résistant en fluage pendant une longue durée à chaud 21 Les fontes

22 Annexes

23 Références. Index

Août 2003. ISBN 2-906 643-27-0. Un volume $17 \times 25 \mathrm{~cm}$ relié, sous couverture cartonnée

416 pages, 413 illustrations, 34 tableaux, 466 références $-85 €+$ port

SIRPE éditeur, 76 rue de Rivoli, F-75004 Paris - Tél. 0142785220 - Fax 0142744048 - E-mail sirpe@noos.fr - Internet: www.sirpe.com 